\title{
EIGENFUNCTION EXPANSIONS AND SCATTERING THEORY FOR PERTURBED ELLIPTIC PARTIAL DIFFERENTIAL OPERATORS ${ }^{1}$
}

\author{
BY PETER C. GREINER
}

Communicated by F. Browder, March 19, 1964

1. A number of papers discussing the spectral decomposition and eigenfunction expansion for partial differential operators appeared in the last few years. Browder [1], [2], [3], [4], Gårding [5] and Mautner [12] proved the existence of an abstract eigenfunction expansion for elliptic partial differential operators. In 1953 A. Ya. Povzner [13] considered the detailed spectral decomposition of $-\Delta+q(x)$. This was completed by $T$. Ikebe [6] who used the theory of wave operators as developed by Kato [8] and Kuroda [10], [11].

In this note we investigate an eigenfunction expansion for the operator $P(D)+q(x)$ where $P(D)$ is a linear homogeneous elliptic partial differential operator with constant coefficients. Detailed proofs of the results will appear elsewhere.

2. The Euclidean $n$-space will be denoted by $R_{n}$ or $M_{n}$ with elements $x=\left(x_{1}, \cdots, x_{n}\right)$ or $k=\left(k_{1}, \cdots, k_{n}\right)$ respectively. $\int f(x) d x$ denotes integration with respect to Lebesgue measure. We set

$$
-D_{j}=\frac{\partial}{i \partial x_{j}} \quad \text { for } 1 \leqq j \leqq n .
$$

Let $P(x)$ be a homogeneous elliptic polynomial, i.e. $P(x) \geqq c|x|^{2 p}$ where $2 p$ is the order of $P(x)$. Then $P(D)=P\left(D_{1}, \cdots, D_{n}\right)$ is a linear homogeneous elliptic partial differential operator. All through this note we assume that $4 p>n$. It is well known that $P(D)$ can be extended to a selfadjoint operator $\widetilde{P}(D)$ in $L_{2}\left(R_{n}\right)$. Let $q(x) \in C_{2[n / 2]}$ with $q(x)=O\left(|x|^{-n-h}\right)$ for some $h>0$. Then by Theorem 1 of [11], $\widetilde{P}(D)+q(x)$ is a selfadjoint operator in $L_{2}\left(R_{n}\right)$. Let $\left\{E_{t}\right\}$ and $\left\{P_{t}\right\}$, $-\infty<t<+\infty$, be the resolutions of the identity for $\widetilde{P}(D)$ and $\widetilde{P}(D)$ $+q(x)$ respectively. Define

1 This research was partially supported by the National Science Foundation under Grant No. NSF-GP-2283. The results reported in this note are contained in a dissertation submitted in partial fulfillment of the requirements for the Ph.D. at Yale University. We are happy to have this opportunity to express our gratitude to Professor Felix E. Browder for his generous advice and many helpful conversations during the preparation of this paper. 


$$
\begin{aligned}
U_{t} & =\exp (i t H) \exp \left(-i t H_{0}\right), \\
W_{ \pm} & =s-\lim U_{t}, \quad t \rightarrow \pm \infty
\end{aligned}
$$

whenever the strong limits exist. $W_{ \pm}$are the wave operators.

For the sake of simplicity we will use the notation $H_{0}=\widetilde{P}(D)$ and $H=H_{0}+Q$ where $Q$ is the operator of multiplication by $q(x)$ in $L_{2}\left(R_{n}\right)$.

Theorem 1. (i) $H=H_{0}+Q$ has pure point spectrum in $(-\infty, 0)$. Furthermore the negative eigenvalues are of finite multiplicity with zero as the only possible limit point.

(ii) For $f(x) \in P_{0} L_{2}\left(R_{n}\right)$ we have the expansion

$$
f(x)=\underset{N \rightarrow \infty}{\operatorname{li} . \mathrm{m} .} \sum_{j=1}^{N} f_{j} u_{j}(x) \text { with } \sum_{j=1}^{\infty}\left|f_{j}\right|^{2}<+\infty
$$

where $\left\{u_{j}, j=1,2, \cdots\right\}$ are the eigenfunctions corresponding to the eigenvalues $h_{j}, j=1,2, \cdots$ in $(-\infty, 0)$ counted according to their multiplicity and

$$
f_{j}=\int_{R_{n}} f(x)\left[u_{j}(x)\right]-d x, \quad j=1,2, \cdots .
$$

(iii) For $f(x) \in D(H) \cap P_{0} L_{2}\left(R_{n}\right), H$ has the diagonal representation

$$
H f(x)=\underset{N \rightarrow \infty}{\text { li.m. }} \sum_{j=1}^{N} h_{j} f_{j} u_{j}(x) .
$$

Definition 1. Let $A$ be a selfadjoint operator in $L_{2}\left(R_{n}\right)$. For $f(x) \in L_{2}\left(R_{n}\right), z \in \Omega$ and $\operatorname{Im} z>0$, where $\Omega=\{z \mid 0<a<\operatorname{Re} z<b<+\infty$, $0 \leqq \operatorname{Im} z \leqq c\}$ for some $a, b$, and $c$, let

$$
\left\{(A-z)^{-1} f\right\}(x)=\int_{R_{n}} K(x-y ; z) f(y) d y
$$

where $K(x ; z)$ is defined for all $z \in \Omega$. We say that $A$ has property $K$, if

$$
K(x ; z)=\frac{V_{z}\left(x_{1}, \cdots, x_{n}\right)}{|x|^{m}}
$$

where $m=(n-1) / 2, V_{z}\left(x_{1}, \cdots, x_{n}\right)$ is continuous in $\Omega \times R_{n}$ and bounded in $R_{n}$, uniformly in $z \in \Omega$.

DEFINITION 2. $s>0$ is a singular point for the operator $H=H_{0}+Q$ if

$$
-f(x)=\int_{R_{n}} K(x-y ; s) q(y) f(y) d y
$$


for some non-null $f \in B$, where $B$ is the Banach space of all continuous functions vanishing at infinity with the sup norm.

Theorem 2. Let $H_{0}=\widetilde{P}(D)$ have property $K$. Let $(a, b), 0<a<b$ $<+\infty$, have no singular points. Then:

(i) $H$ has no eigenvalues in $(a, b)$. Moreover, the spectrum of $H$ in $(a, b)$ is absolutely continuous.

(ii) For $f \in\left(P_{b}-P_{a}\right) L_{2}\left(R_{n}\right)$, we can define the generalized Fourier transform $f^{\wedge}(k) \in L_{2}\left(M_{n}\right)$ of $f$ as follows

$$
f^{\wedge}(k)=(2 \pi)^{-n / 2} \text { l.i.m. } \int_{R_{n}}[F(x, k)]-f(x) d x
$$

where $F(x, k)$ is uniformly continuous and bounded on $R_{n} \times M_{n} . f^{\wedge}(k)$ $=0$ if $P(k) \notin(a, b)$. Furthermore $f^{\wedge}(k) \in L_{2}\left(M_{n}\right)$ with $\left\|f^{\wedge}\right\|=\|f\|$.

(iii) For $f \in\left(P_{b}-P_{a}\right) L_{2}\left(R_{n}\right)$ we have

$$
f(x)=(2 \pi)^{-n / 2} \int_{a<P(k)<b} F(x, k) f^{\wedge}(k) d k .
$$

(iv) For $f \in\left(P_{b}-P_{a}\right) L_{2}\left(R_{n}\right)$ we have the diagonal representation for $H$

$$
H f(x)=(2 \pi)^{-n / 2} \int_{a<P(k)<b} P(k) F(x, k) f^{\wedge}(k) d k .
$$

(v) For every $k \in M_{n}$ with $a<P(k)<b$

$$
P(D) F(x, k)+q(x) F(x, k)=P(k) F(x, k) .
$$

Part (v) of Theorem 2 shows that the spectral decomposition we obtained is actually an eigenfunction expansion. This is clear if we note that $F(x, k)$ is a weak solution of the elliptic partial differential equation

$$
P(D) u(x)+q(x) u(x)-P(k) u(x)=0
$$

(cf. F. John [7]).

The following result is suggested by Theorem 2 .

THEOREM 3. $H$ acting in $\left(P_{b}-P_{a}\right) L_{2}\left(R_{n}\right)$ is unitarily equivalent to $H_{0}$ acting in $\left(E_{b}-E_{a}\right) L_{2}\left(R_{n}\right)$. To be precise we have

$$
H=\left[W_{-}\left(E_{b}-E_{a}\right)\right] H_{0}\left[W_{-}\left(E_{b}-E_{a}\right)\right]^{*}
$$

where * denotes the inverse of a partially isometric operator.

Next we show if $q(x)$ is sufficiently restricted $\widetilde{P}(D)+Q$ has no singular points in $(0,+\infty)$. 
TheOREM 4. Let $\widetilde{P}(D)$ have property $K$. Let $q(x)$ satisfy the inequality

$$
|q(x)| \leqq \begin{cases}0 & \text { for }|x|<R_{0}, \\ C_{0}|x|^{-n-1} & \text { for }|x|>R_{0},\end{cases}
$$

where $C_{0} C^{\prime}<1$ if $\left|V_{\boldsymbol{x}}\left(x_{1}, \cdots, x_{n}\right)\right|<C^{\prime}$ and $R_{0}>\sup \left(2 \omega_{n}, 2\right)$ with $\omega_{n}$ designating the surface area of the unit sphere in $R_{n}$. Then $H=H_{0}+Q$ has $(0,+\infty)$ as its absolutely continuous spectrum.

This is proved by assuming that (s) has a solution $f \in B$. Then we estimate the integral and obtain a uniform bound on $f(x)$, which when repeated shows that $f(x) \equiv 0$.

To investigate the singular points in the rest of this note we restrict the discussion to $P(D)=\left(D_{1}^{2}+\cdots+D_{n}^{2}\right)^{p}=(-\Delta)^{p}$ in $R_{2 m+1}$ for $m=1,2, \cdots$ and $4 p>2 m+1$. Using the fact that $K(x ; z) \in L_{2}\left(R_{2 m+1}\right)$ if $\operatorname{Im} z>0$ we obtain

TheOREM 5. For $z \in \Omega$ with $\operatorname{Im} z>0$ we have:

(i) For $r>0$ and $d=1,2, \cdots, p-1$ $(-\Delta)_{x}^{d} K\left(x-y ; r^{2 p}\right)=-\frac{(-i)^{m+1} \pi}{p(2 \pi)^{m+1}} r^{2 d} \frac{r^{m+1-2 p} e^{i r|x-y|}}{|x-y|^{m}}+O\left(|x|^{-m-1}\right)$,

(ii) $(-\tilde{\Delta})^{p}$ has property $K$.

Lemma. Let $f \in B$ be a solution of

$$
f(x)=-\int_{R_{2 m+1}} K\left(x-y ; r^{2 p}\right) q(y) f(y) d y .
$$

Then

$$
\begin{aligned}
f(x)= & \frac{\pi(-i)^{m+1} \gamma^{m+1-2 p}}{p(2 \pi)^{m+1}} \frac{e^{i r|x|}}{|x|^{m}} \int_{R_{2 m+1}} e^{i r(x /|x|, y)} q(y) f(y) d y \\
& +O\left(|x|^{-m-1 / 2-h / 2}\right)+O\left(|x|^{-m-1}\right) .
\end{aligned}
$$

Moreover

$$
\frac{d}{d|x|}(-\Delta)^{a} f(x)=(i r)^{2 q+1} f(x)+O\left(|x|^{-m-h / 2}\right)+O\left(|x|^{-1-m}\right) .
$$

Using this lemma and Green's theorem we obtain

THEOREM 6. Let $f \in B$ be a solution of the homogeneous integral equation $(\mathrm{r})$. Then $f(x)$ is an $L_{2}$ eigenfunction of $H=(-\Delta)^{p}+q(x)$.

The above lemma generalizes the well-known "radiation" condition 
of Sommerfeld. Now Theorems 1 and 2 give us the complete description of $H=(-\tilde{\Delta})^{p}+q(x)$ acting in $\left(I-P_{0}\right) L_{2}\left(R_{2 m+1}\right)$.

Theorem 7. On the positive real axis, $H$ has absolutely continuous spectrum and eigenvalues. The point spectrum is a closed, denumerable, nowhere dense set.

\section{BIBLIOGRAPHY}

1. F. E. Browder, Eigenfunction expansions for formally selfadjoint partial differential operators. I, Proc. Nat. Acad. Sci. U.S.A. 42 (1956), 769-771.

2. - Eigenfunction expansions for formally selfadjoint partial differential operators. II, Proc. Nat. Acad. Sci. U.S.A. 42 (1956), 870-872.

3. - The eigenfunction expansion theorem for the general selfadjoint elliptic partial differential operator. I, Proc. Nat. Acad. Sci. U.S.A. 40 (1954), 454-459.

4. - The eigenfunction expansion theorem for the general selfadjoint elliptic partial differential operator. II, Proc. Nat. Acad. Sci. U.S.A. 40 (1954), 459-463.

5. L. Gårding, Eigenfunction expansions connected with elliptic differential operators, Tolfte Skandinaviska Matematikerkongressen, Lund, 1953, pp. 44-56.

6. T. Ikebe, Eigenfunction expansions associated with the Schroedinger operators and their applications to scattering theory, Arch. Rational Mech. Anal. 5 (1960), 1-34.

7. F. John, Plane waves and partial differential equations, Interscience, New York, 1955.

8. T. Kato, On finite dimensional perturbations of self-adjoint operators, J. Math. Soc. Japan 9 (1957), 239-249.

9. - Fundamental properties of Hamiltonian operators of Schroedinger type, Trans. Amer. Math. Soc. 70 (1951), 195-211.

10. S. T. Kuroda, On the existence and unitary property of the scattering operator, Nuovo Cimento 12 (1959), 431-454.

11. - Perturbation of continuous spectra by unbounded operators. I, J. Math. Soc. Japan 11 (1959), 247-262.

12. F. I. Mautner, On eigenfunction expansions, Proc. Nat. Acad. Sci. U.S.A. 30 (1953), 49-53.

13. A. Ya. Povzner, On the expansions of arbitrary functions in terms of the eigenfunctions of the operator $-\Delta u+c u$, Mat. Sb. (N. S.) 32 (74) (1953), 109-156. (Russian)

YALE UNIVERSITY 